\title{
DÜBLIN
}

Technological University Dublin

ARROW@TU Dublin

2006-06-01

\section{Gauging VolP Call Quality from 802.11 WLAN Resource Usage}

Miroslaw Narbutt

Technological University Dublin, miroslaw.narbutt@tudublin.ie

Mark Davis

Technological University Dublin, mark.davis@tudublin.ie

Follow this and additional works at: https://arrow.tudublin.ie/commcon

Part of the Electrical and Electronics Commons

\section{Recommended Citation}

Narbutt, M. \& Davis, M. (2006) Gauging VolP call quality from 802.11 WLAN resource usage. IEEE International Symposium on a World of Wireless, Mobile and Multimedia Networks (WoWMoM06), Buffalo, New York, USA, June, 2006.

This Conference Paper is brought to you for free and open access by the Communications Network Research Institute at ARROW@TU Dublin. It has been accepted for inclusion in Conference papers by an authorized administrator of ARROW@TU Dublin. For more information, please contact arrow.admin@tudublin.ie, aisling.coyne@tudublin.ie,gerard.connolly@tudublin.ie.

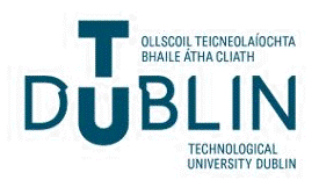




\title{
Gauging VoIP call quality from 802.11 WLAN resource usage
}

\author{
Miroslaw Narbutt and Mark Davis \\ Communications Network Research Institute \\ School of Electronic and Communications Engineering \\ Dublin Institute of Technology, IRELAND \\ narbutt@cnri.dit.ie,mark.davis@dit.ie
}

\begin{abstract}
In this paper we experimentally study the relationship between resource utilization in the wireless LAN and the quality of VoIP calls transmitted over the wireless medium. Specifically we evaluate how its overall capacity is shared between three basic MAC bandwidth components (load, access, and free) as the number of VoIP calls increases and how it influences transmission impairments (delay, loss, and jitter) and thus call quality. Resource utilization (under the MAC bandwidth components framework) is calculated by a WLAN probe application that passively "sniffs" packets at the L2/MAC layer of the wireless medium and analyses their headers and temporal characteristics. The quality of VoIP calls is predicted using an extended version of the ITU-T E-model, which estimates user satisfaction from time varying transmission impairments. Through experimentation with various codecs and packetization schemes we found that as the load (number of calls) reaches the available capacity level, packet delays and jitter increase dramatically resulting in the call quality becoming degraded. We show how these MAC bandwidth components maybe used to assess the VoIP call quality on 802.11 WLANs.
\end{abstract}

\section{Introduction}

Wireless VoIP applications are resource hungry. When the effective available bandwidth in a 802.11 WLAN is too low establishing a new call in addition to the ongoing calls can have disastrous consequences. Sometimes call quality can become unacceptable for all ongoing calls. In this paper we experimentally study the relationship between resource utilization in the wireless LAN and the quality of VoIP calls transmitted over the wireless medium. Specifically we evaluate how its overall capacity is shared between basic bandwidth components (load, access, and free) as the number of VoIP calls increases and how it influences transmission impairments (delay, loss, and jitter) and thus call quality. We believe that this type of resource information that could be useful for potential QoS provisioning and call admission schemes.

This paper is structured as follows: In Section 2 we describe a method for real-time monitoring resource utilization in an 802.11 WLAN. In Section 3 we introduce a method for predicting VoIP call quality based on transmission impairments. In Section 4 we show results of our experiments and we discuss the relationship between resource utilization and the quality of VoIP calls transmitted over the wireless medium. Finally, we present the conclusion.

\section{Resource utilization monitoring in 802.11 WLAN}

It is possible to distinguish two basic time intervals on the wireless medium: busy and idle. The busy intervals represent the time during which wireless devices transmit data/managements frames and their positive acknowledgments. The complementary intervals are idle intervals where the wireless medium is silent. These idle intervals are fundamental to the operation of the 802.11 MAC protocol as the backoff mechanism requires an idle medium in order to decrement the backoff counters having first deferred for a period of DIFS (i.e. 50us in 802.11b). Wireless devices with a frame to transmit use these idle intervals in order to win transmission opportunities for the frame. Typically, the time required to win a transmission opportunity, i.e. the access time comprises of two components: a time deferring for DIFS following the medium becoming idle and a time spent decrementing its backoff counter, which is slotted (where $T_{\text {slot }}=20 \mu s$ in $802.11 \mathrm{~b}$ ). However, if 
a wireless device does not have a frame to transmit, then these idle intervals represent unused or free time on the medium. This free time constitutes spare capacity on the medium in the sense that it can be utilized to win additional transmission opportunities for the device if required. Figure 1 shows these various time intervals of interest.

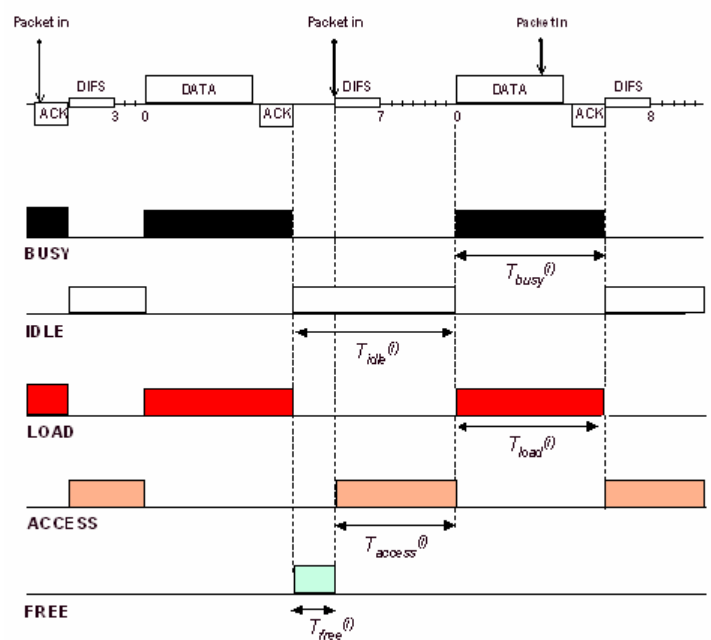

Figure 1. Time intervals involved in accessing the medium

$T_{\text {busy }}(i)$ are the busy time intervals on the medium when the wireless devices are transmitting their frames, $T_{\text {idle }}(i)$ are the times when the medium is not busy and comprises access time intervals $T_{\text {access }}(i)$ when a device actively contend for transmission opportunities and $T_{\text {free }}(i)$ which represent the unused idle time (i.e. available capacity).

The time intervals involved in accessing the wireless medium are closely related to the MAC bandwidth components we are introducing to characterize WLAN resource utilization. Consequently we distinguish three basic MAC bandwidth components:

- a load bandwidth ( $\left.B W_{\text {load }}\right)$ that is associated with the transmission of the data frames

- an access bandwidth ( $\left.B W_{\text {access }}\right)$ that is associated with the contention mechanism (whereby a wireless device wins access to the wireless medium)

- a free bandwidth ( $B W_{\text {free }}$ ) that corresponds to the remaining unused idle time that can be viewed as spare or available capacity
By examining the MAC packet headers, it is possible to identify the sender of a frame, the busy time on the medium used by that device in transmitting its load:

$$
T_{\text {load }}(k)=\sum_{i} T_{\text {load }}{ }^{(k)}(i)
$$

This busy time can be converted into bandwidth load of the device:

$$
B W_{\text {load }}(k)=\frac{T_{\text {load }}(k)}{T_{\text {busy }}+T_{\text {idle }}} \times \text { Line _ Rate }
$$

The busy and idle time intervals are summed over the interval of interest as follows:

$$
\begin{aligned}
& T_{\text {busy }}=\sum_{i} T_{\text {busy }}{ }^{(i)} \\
& T_{\text {idle }}=\sum_{i} T_{\text {idle }}{ }^{(i)}
\end{aligned}
$$

where $T_{\text {busy }}{ }^{(i)}$ and $T_{\text {idle }}{ }^{(i)}$ are the durations of the $i^{\text {th }}$ busy and idle intervals respectively within the interval of interest.

The load bandwidth associated with a particular wireless device $B W_{\text {load }}(k)$ is directly related to the throughout of the device. The amount of free bandwidth $B W_{\text {free }}(k)$ is directly related to the level of QoS achieved while transmitting its traffic load, where the greater the free capacity available, the better the QoS likely to be experienced (Figure 2).

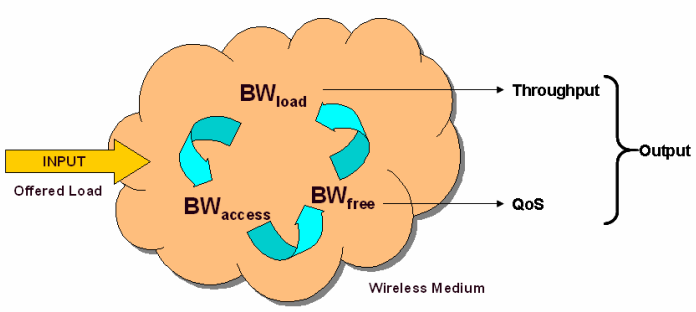

Figure 2. Mac bandwidth components

The busy bandwidth BWbusy is the sum of the BWload overall stations corrected by the amount of bandwidth lost due to collisions between devices attempting to transmit at the same time: 


$$
B W_{\text {busy }}=\sum_{k} B W_{\text {load }}(k)-B W_{\text {collisions }}
$$

The access bandwidth $B W_{\text {access }}(k)$ is obtained by inferring the average access time $T_{\text {access }}(k)$ from a statistical characterization of the idle interval and the load intervals of the device. The resulting access bandwidth can be calculated as:

$$
B W_{\text {access }}(k)=\frac{T_{\text {access }}(k)}{T_{\text {busy }}+T_{\text {idle }}} \times \text { Line_Rate }
$$

The free bandwidth $B W_{\text {free }}(k)$ can be derived from the following formula:

$$
B W_{\text {busy }}+B W_{\text {access }}(k)+B W_{\text {free }}(k)=\text { Line_Rate }
$$

A WLAN resource monitoring application based upon this MAC bandwidth components framework is described in $[1,2]$. It non-intrusively measures the bandwidth utilization in real-time on a per-station basis. The application has shown to be particularly effective in characterizing WLAN resource usage in the context of video streaming $[3,4,5]$. This time it is being used in the context of real-time VoIP transmission in a WLAN.

\section{Predicting VoIP call quality}

\subsection{ITU-T E-model - transmission planning tool}

A tool that can be used to predict user satisfaction of a conversational speech quality is the ITU-T E-model. The E-Model was originally developed by ETSI [6] as a transmission planning tool, and then standardized by the ITU as G.107 [7] and suggested by TIA [8] as "a tool that can estimate the end-to-end voice quality, taking the IP telephony parameters and impairments into account". This method combines individual impairments (loss, delay, echo, codec type, noise, etc.) due to both the signal's properties and the network characteristics into a single R-rating.

The transmission rating factor $\mathrm{R}$ can lie in the range from 0 to 100: high values of $\mathrm{R}$ in a range of $90<\mathrm{R}<$ 100 should be interpreted as excellent quality, while a lower value of $\mathrm{R}$ indicates a lower quality. Values below 50 are clearly unacceptable and values above
94.15 are unobtainable in narrowband telephony. The rating factor $\mathrm{R}$ is a linear combination of the individual impairments:

$$
R=\left(R_{o}-I_{s}\right)-I_{d}-I_{e}+A
$$

In the context of VoIP transmission assessment, the delay impairment $I_{d}$ and equipment impairment $I_{e}$ (which captures the effect of information loss due to encoding scheme and packet loss) are relevant. The other impairments - loud connection and quantization impairment $I_{S}$, basic signal to noise ratio $R_{0}$ and the "advantage factor" $A$ do not depend on the transmission over the network. Since values of $\mathrm{R}$ above 94.15 are unobtainable in narrowband (300 to $3400 \mathrm{~Hz}$ ) telephony, we can write the $\mathrm{R}$ rating for $\mathrm{G} .711$ audio as:

$$
R=94.15-I_{d}-I_{e}
$$

As a general rule, the perceived quality decreases with increasing delay and/or increasing level of the received echo signal but listener echo can be neglected if there is sufficient control of the talker echo. The degree of annoyance of talker echo depends on the level difference between the original voice and the received echo signal. This level difference is characterized by so-called "Talker Echo Loudness Rating” (TELR). ITU-T Recommendation G.131 provides useful information regarding talker echo as a parameter by itself [9].

The relation between delay impairment $I_{d}$ and mouth-to-ear delay for five values of TELR is shown in Figure 3 [11].

Equipment impairment factor $I_{e}$ captures effects of information loss, due to both encoding scheme and packet loss (including late packet arrival). ITU-T Recommendation G.113 [10] gives detailed values of this impairment factor for various codecs as a function of packet loss.

Figure 4 show for several codecs (and PLC techniques) how the equipment impairment increases as packet loss increases. Figure 4 shows how equipment impairment $I_{e}$ increases as packet loss increases considering several codecs (and PLC techniques). 


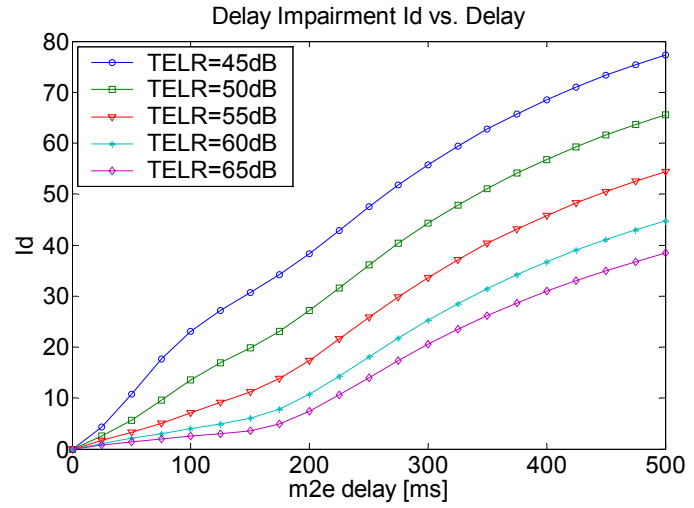

Figure 3 Delay impairment as a function of the one-way delay (based on $\mathbf{R}$ values specified in

[11])

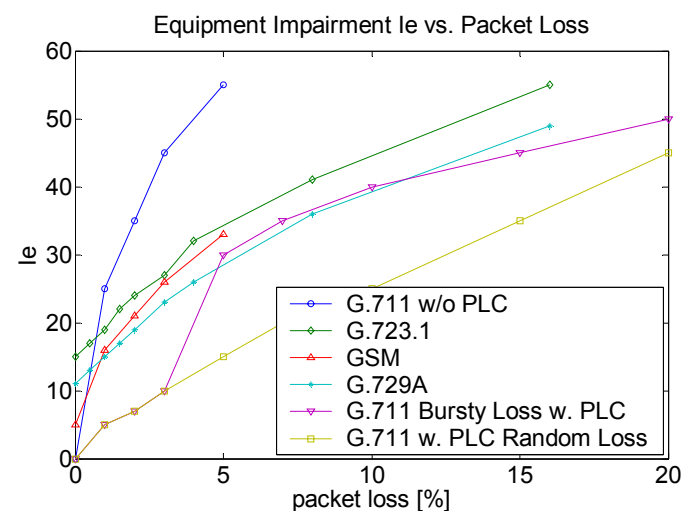

Figure 4 Equipment impairment as a function of the packet (based on values specified in [13]).

If the mouth-to-ear delay, echo loss, encoding scheme, and packet loss are known, the quality of a conversational speech transmitted over IP can be predicted as shown in Figure 5.

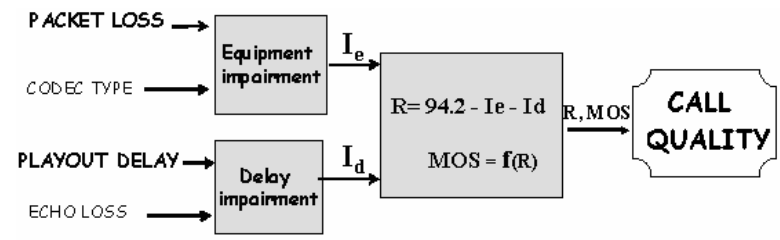

Figure 5. Predicting VoIP quality using the Emodel methodology.

\subsection{Assessing time varying quality of the call}

The E-model does not take onto account the dynamics of a transmission but relies on static transmission parameters. A natural approach is to divide the call duration into fixed time intervals and assess the call quality of each interval independently. This method for assessing time-varying quality of a call was proposed in $[11,12]$. There is however one important parameter (not mentioned by authors) that influences these calculations, namely the time interval for which the average playout delay and the average loss is calculated. Following [13] we assumed that the time window of 10 seconds is sufficient because it is within the recommended length for PESQ algorithm. Playout buffer module calculates playout delays and resulting packet loss according to a specific playout buffer algorithm. Then average mouth-to-ear delay and average packet loss (due to both late packet arrival and network loss) is obtained for every 10 seconds of a transmission as shown in Figure 6. The corresponding delay impairments (assuming given echo loss), equipment impairments (assuming given codec type), and the resulting rating factor $\mathrm{R}$ are calculated using Emodel formulas as shown in Figure 7.
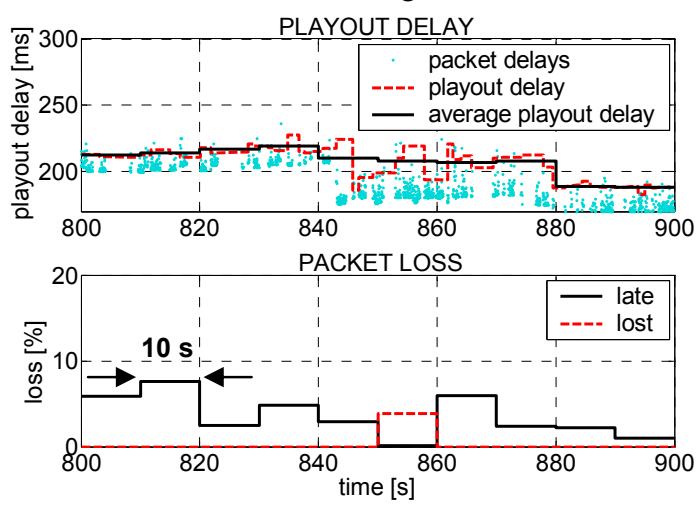

Figure 6. Average playout delays and packet loss for each 10 seconds of a call DELAY IMPAIRMENT
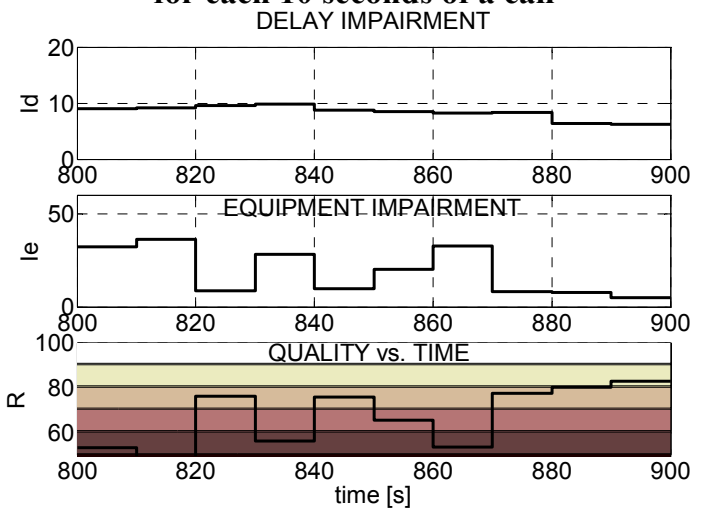

Figure 7. Corresponding transmission impairments and time varying quality of a call (rating $R$ )

\subsection{Assessing user satisfaction}

ITU-T Recommendation G.109 [14] introduces categories of user satisfaction based on the transmission rating factor $\mathrm{R}$. The definitions of those 
categories in terms of ranges of $\mathrm{R}$ are found in Table 1 . Also provided is the relation between $\mathrm{R}$ and the MOS score.

\begin{tabular}{|c|c|c|}
\hline $\mathbf{R}$ & MOS & User satisfaction \\
\hline $90-94.5$ & $4.34-4.50$ & very satisfied \\
\hline $80-90$ & $4.03-4.34$ & satisfied \\
\hline $70-80$ & $3.60-4.03$ & some users dissatisfied \\
\hline $60-70$ & $3.10-3.60$ & many users dissatisfied \\
\hline $50-60$ & $2.58-3.10$ & nearly all users dissatisfied \\
\hline $0-50$ & $1.00-2.58$ & not recommended \\
\hline
\end{tabular}

Table 1: Definition of categories of user satisfaction [17]

Using the formula in equation (9) we created contours of quality as a function of delay and loss. Such quality contours determine the rating factor $\mathrm{R}$ for all possible combinations of loss and delay, with their shape being determined by both impairments $I_{d}$ and $I_{e}$. They give a measure of the impact of packet loss and compression scheme on speech quality and the effect of delay and echo on interactive conversations. Figure 10 shows those quality planes for G.711 encoding scheme (assuming bursty loss of packets) and for five different echo loss levels (TELR $=45,50$, $55,60,65 \mathrm{~dB})$. Of a particular interest here is the ability to find different combinations of loss and delay that results in the same user satisfaction.
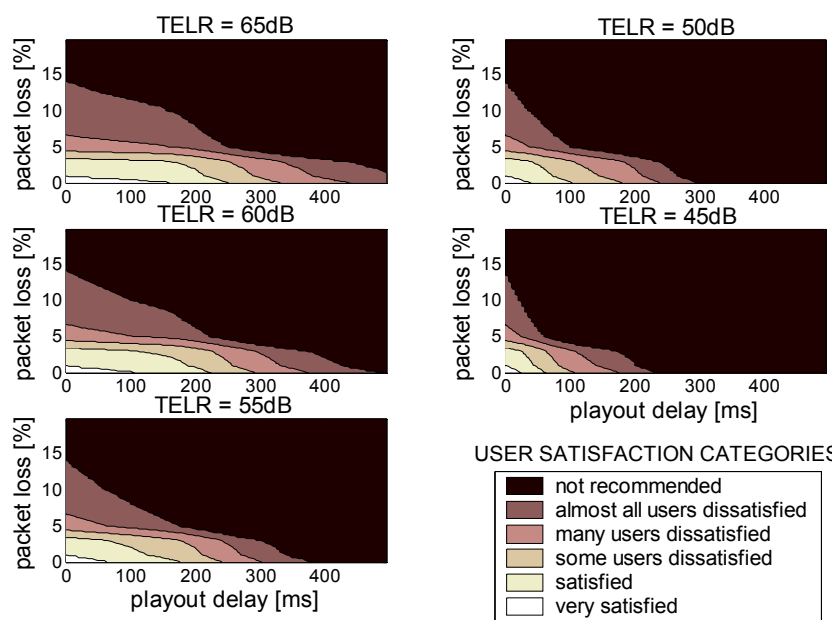

USER SATISFACTION CATEGORIES:

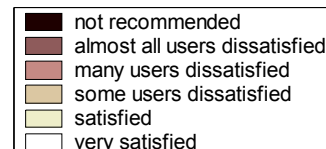

Figure 8. Quality contours for conversational speech for different echo levels (calculated for G.711 w. PLC and bursty loss)
The procedure of assessing overall user satisfaction with the use of quality contours is described below and illustrated in Figures 9 and 10 The playout buffer module calculates playout delays and resulting packet loss with the use of a specific playout algorithm. Quality contours are chosen for a specific encoding scheme and echo cancellation level. layout delays and packet losses are mapped on a chosen quality contours. Distribution of playout delays and packet losses on quality contours can be used to assess end-to-end conversational voice quality as perceived by an average user. Overal user satisfaction can be obtained from a pie chart that is directly related to distribution of playout delays on quality contours.

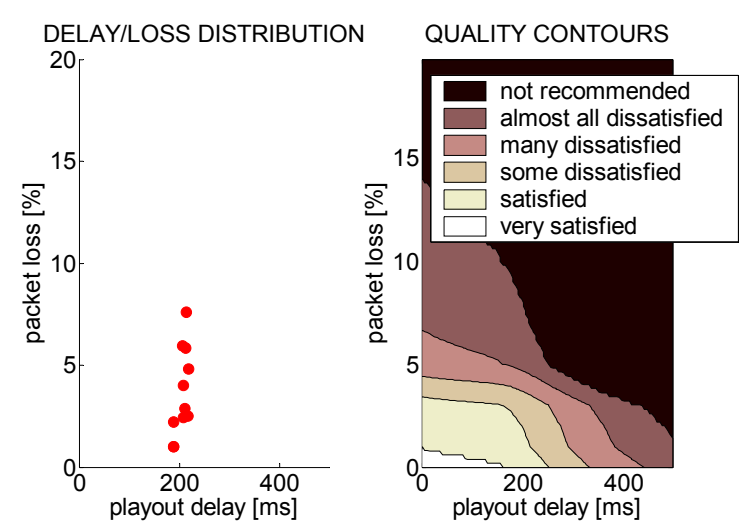

Figure 9. Calculated playout delays and packet loss to be mapped to a specific quality contours

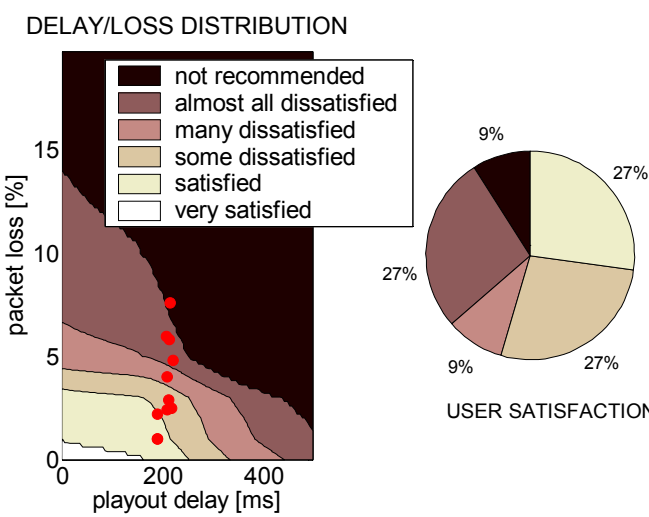

Figure 10 Distribution of playout delays and packets loss on quality contours (codec G.711 w. PLC, bursty loss, echo level TELR = 45dB) and resulting user satisfaction 
As shown in Figure 12, using the specific algorithm, with the specific codec and the specific echo loss: an average user would be satisfied $27 \%$ of the time, some users could be dissatisfied $27 \%$ of the time, many users would be dissatisfied $9 \%$ of the time, almost all users would be dissatisfied $27 \%$ of the time, during $9 \%$ of the time quality was not acceptable at all. This method of predicting user satisfaction from time varying transmission impairments has shown to be particularly effective in evaluating various playout buffer algorithms $[15,16]$ and assessing audio codecs performance in Voice over WLAN systems [17].

\section{Experimental results}

\subsection{Experimental testbed and testing scenario.}

The $802.11 \mathrm{~b}$ wireless/wired testbed consists of 16 desktop PCs acting as wireless VoIP terminals and one desktop PC acting as an access point (AP). All machines in the testbed use the $802.11 \mathrm{~b}$ MPCMCI wireless cards based on Atheros chipsets controlled by MadWiFi wireless drivers and Linux OS (kernel 2.6.9). All of the nodes are also equipped with a $100 \mathrm{MBps}$ wired Ethernet. A machine that acts as an access point routes between the wired and the wireless networks allowing for bi-directional traffic wired-towireless and vice-versa (each machine has two interfaces: one on the wireless and one on wired side). During experiments each VoIP terminal runs one VoIP and all sessions are bi-directional. This way each terminal acts as the source of an uplink flow and the sink of a downlink for VoIP session.

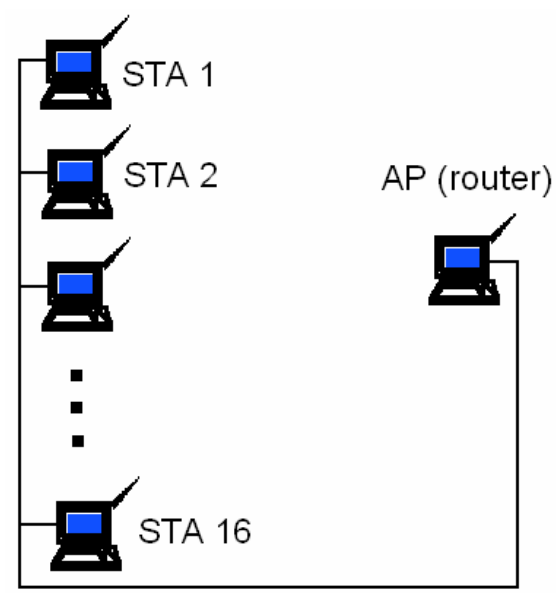

Figure 11. Experimental 802.11b testbed
The wireless stations are located within 5 meters range from the AP to ensure that the wireless link quality is good. This testbed is illustrated in Figure 11.

Voice traffic was generated using RTPtools [18]. Three codecs and various packetization schemes were considered:

- G.711:

$$
\begin{array}{cl}
\circ & 80 \text { bytes } / 10 \mathrm{~ms} \text { payload, } \\
\circ & 160 \text { bytes } / 20 \mathrm{~ms} \text { payload, } \\
\circ & 240 \text { bytes } / 30 \mathrm{~ms} \text { payload, }
\end{array}
$$

- G.723.1 :

○ 24/bytes30ms payload,

- G.729:

$$
\begin{aligned}
& \text { 10bytes } / 10 \mathrm{~ms} \text { payload, } \\
& \circ \text { 20bytes } / 20 \mathrm{~ms} \text { payload, } \\
& \circ \quad 30 \text { byes } / 30 \mathrm{~ms} \text { payload. }
\end{aligned}
$$

During experiments we used two types of voice traffic:

- CBR

- Bidirectional ON-OFF (alternating active and passive periods in accordance with [19])

The duration of each experiment was one hour long during which time all experimental data (packet arrival times, timestamps, sequence numbers, and marker bits) were collected at the receiving terminal and processed later (off-line) with a program that simulated the behavior of the basic adaptive playout algorithm [20]. The main objective of the experiments was to evaluate how overall capacity of the wireless medium is shared between three basic MAC bandwidth components (load, access, and free) as the number of VoIP calls increases and how it influences VoIP call quality. For that reason we were successively establishing new calls in addition to the ongoing calls. Figure 12 illustrates testing scenario of the experiments.

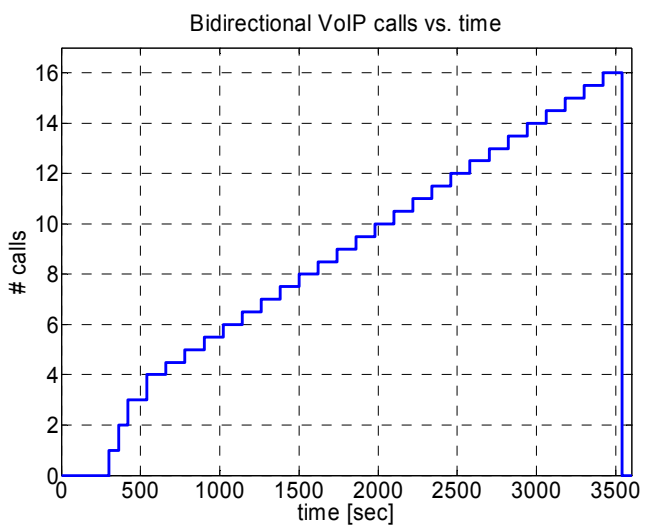

Figure 12. Testing scenario 


\section{Wireless measurements}

Resource utilization (in the form of three MAC bandwidth components) was measured during experiments by a WLAN probe application as it was described in Chapter 2. The quality of VoIP calls was predicted after each experiment based on collected transmission impairments as it was described in Section 3. Figures 13-36 show how overall capacity of the wireless medium was shared between three basic MAC bandwidth components (load, access, and free) as the number of VoIP calls was increasing and how it influenced transmission impairments (delay, loss, and jitter) and thus call quality and overall user satisfaction. Rating factor $\mathrm{R}$ that represents predicted call quality and MAC bandwidth components are summarized in Table 2 and Table 3 for CBR and ONOFF traffic types respectively.

\begin{tabular}{|c|c|c|c|c|}
\hline CBR & $\begin{array}{c}\text { QUALITY } \\
\text { "wired-to- } \\
\text { wireless" }\end{array}$ & \multicolumn{3}{|c|}{$\begin{array}{c}\text { MAC BANDWIDTH } \\
\text { COMPONENTS (AP) }\end{array}$} \\
\hline & R & LOAD & ACCESS & FREE \\
\hline $\begin{array}{c}\text { G.711 } \\
10 \mathrm{~ms}\end{array}$ & 52 & $49 \%$ & $31 \%$ & $20 \%$ \\
\hline $\begin{array}{c}\text { G.711 } \\
20 \mathrm{~ms}\end{array}$ & 78 & $31 \%$ & $21 \%$ & $48 \%$ \\
\hline $\begin{array}{c}\text { G.711 } \\
30 \mathrm{~ms}\end{array}$ & 76 & $24 \%$ & $12 \%$ & $64 \%$ \\
\hline $\begin{array}{c}\text { G.723.1 } \\
30 \mathrm{~ms}\end{array}$ & 75 & $15 \%$ & $12 \%$ & $73 \%$ \\
\hline $\begin{array}{c}\text { G.729 } \\
10 \mathrm{~ms}\end{array}$ & 62 & $41 \%$ & $36 \%$ & $23 \%$ \\
\hline $\begin{array}{c}\text { G.729 } \\
20 \mathrm{~ms}\end{array}$ & 77 & $22 \%$ & $19 \%$ & $59 \%$ \\
\hline $\begin{array}{c}\text { G.729 } \\
30 \mathrm{~ms}\end{array}$ & 74 & $15 \%$ & $12 \%$ & $73 \%$ \\
\hline
\end{tabular}

Table 2. Relationship between VolP call quality perceived at the wireless side and Access Point bandwidth utilization (CBR traffic).

From Table 1 it can be seen that that call quality is highly influenced by the amount of free bandwidth at the access point (AP). Of particular interest here is "bandwidth consuming" G.711/10ms encoding scheme. Low level of average free bandwidth (20\%) results with poor call quality (rating factor $\mathrm{R}=52$ ). Similar situation is with "efficient" G.729/10ms encoding (free bandwidth $23 \%$, rating factor $\mathrm{R}=62$ ). This can be explained as follow: Although each of the sixteen VoIP terminals had data to transmit very infrequently (minimal sending interval was $10 \mathrm{~ms}$ ), the access point was sending $n$ times the load downstream to the clients. As a result, for CBR $10 \mathrm{~ms}$ voice packets, only 8 connections can be supported by a single access point. This can be clearly seen in Figures 13, 14 and 31,32. As soon as the eighth VoIP call was placed, jitter and packet delays increased dramatically resulting in the call quality becoming degraded.

\begin{tabular}{|l|c|c|c|c|}
\hline $\begin{array}{l}\text { ON- } \\
\text { OFF }\end{array}$ & $\begin{array}{c}\text { QUALITY } \\
\text { "wired-to- } \\
\text { wireless" }\end{array}$ & \multicolumn{3}{|c|}{$\begin{array}{l}\text { MAC BANDWIDTH } \\
\text { COMPONENTS (AP) }\end{array}$} \\
\hline $\begin{array}{c}\text { av. R } \\
\text { G.711 } \\
10 \mathrm{~ms}\end{array}$ & 79 & $24 \%$ & $19 \%$ & $57 \%$ \\
\hline $\begin{array}{c}\text { G.711 } \\
20 \mathrm{~ms}\end{array}$ & 76 & $16 \%$ & $9 \%$ & $75 \%$ \\
\hline $\begin{array}{c}\text { G.711 } \\
30 \mathrm{~ms}\end{array}$ & 73 & $12 \%$ & $6 \%$ & $82 \%$ \\
\hline $\begin{array}{c}\text { G.723.1 } \\
30 \mathrm{~ms}\end{array}$ & 74 & $8 \%$ & $6 \%$ & $86 \%$ \\
\hline $\begin{array}{c}\text { G.729 } \\
10 \mathrm{~ms}\end{array}$ & 80 & $21 \%$ & $19 \%$ & $60 \%$ \\
\hline $\begin{array}{c}\text { G.729 } \\
20 \mathrm{~ms}\end{array}$ & 77 & $11 \%$ & $9 \%$ & $80 \%$ \\
\hline $\begin{array}{c}\text { G.729 } \\
30 \mathrm{~ms}\end{array}$ & 74 & $8 \%$ & $6 \%$ & $87 \%$ \\
\hline
\end{tabular}

Table 3. Relationship between VolP call quality perceived at the wireless side and Access Point bandwidth utilization (CBR traffic).

With ON-OFF traffic type, the voice activity is $42.6 \%$ according to [19]. In fact we observed that with this traffic type, bandwidth load decreased from $49 \%$ down to $24 \%$ for G.711/10ms and from $41 \%$ down to $21 \%$ for G.729/10ms. As a result the effective available bandwidth increased to $57 \%$ and $60 \%$ resulting with better call quality (Table 3 ).

\section{Conclusions}

Establishing a new call in $802.11 \mathrm{~b}$ WLAN in addition to the ongoing calls can have disastrous consequences. Through experimentation with various codecs and packetization schemes we found close relationship between call quality and wireless resource utilization. When the effective available bandwidth is too low the call quality can become unacceptable for all ongoing calls. Resource utilization can be now monitored by a WLAN probe application that passively "sniffs" packets at the L2/MAC layer of the wireless medium and provides information about three MAC bandwidth components (load, access, and free bandwidth). This is the type of resource information 
that can be required for potential QoS provisioning and call admission schemes.

\section{References}

[1 ] M. Davis, "A Wireless Traffic Probe for Radio Resource Management and QoS Provisioning in IEEE 802.11 WLANs", Proc of the ACM Symposium on Modeling, Analysis and Simlulation of Wireless and Mobile Systems (ACM MSWiM 2004), Venice, Italy, October, 2004

[2] M. Davis and T. Raimondi, "A Novel Framework for Radio Resource Management in IEEE 802.11 Wireless LANs", Proc of the 3rd Intl. Symposium on Modeling and Optimization in Mobile, Ad Hoc, and Wireless Networks (WiOpt 2005), Trentino, Italy, April, 2005

[3] Nicola Cranley and Mark Davis, "Performance Evaluation of Video Streaming with Background Traffic over IEEE 802.11WLAN Networks", Proc of the ACM Workshop on Wireless Multimedia Networking and Performance Modeling (WMuNep'05), October 2005

[4] Nicola Cranley, Mark Davis, "Performance Evaluation of Resource Usage for Unicast Video Streaming over IEEE 802.11 WLAN Networks", Proc. of the 5th Workshop on Applications and Services in Wireless Networks (ASWN 2005), Paris, July 2005

[5] Nicola Cranley, Mark Davis, "Performance Analysis of Network-level QoS with Encoding Configurations for Unicast Video Streaming over IEEE 802.11 WLAN Networks", WirelessCom 2005, Maui, Hawaii, June 2005

[6] N. O. Johannesson: "The ETSI Computation Model: A Tool for Transmission Planning of Telephone Networks", IEEE Communications Magazine, pp. 70-79, January '97

[7] ITU-T Rec. G.107, "The E-Model, a computational model for use in transmission planning”, March, '03

[8] Telecommunications Industry Association, "Voice Quality Recommendations for IP Telephony TIA/EIA/TSB116”, ‘01

[9] ITU-T Rec. G.131, "Talker echo and its control", November '03

[10] ITU-T G.113 Recommendation Appendix I, "Provisional Planning Values for the Equipment Impairment Factor Ie”, October '01

[11] A. P. Markopoulou, PhD Dissertation: "Assessing the Quality of Multimedia Communications over Internet Backbone Networks", Stanford University, 2003

[12] A. P. Markopouolu, F. A. Tobagi, M. J. Karam, "Assessment of VoIP Quality over Internet Backbones", Proceedings of the IEEE Infocom, June, '02
[13] L. Sun, E. C. Ifeachor, "Prediction of Perceived Conversational Speech Quality and Effects of Playout Buffer Algorithms", Proceedings of the IEEE ICC, May, '03

[14] ITU-T Recommendation G.109, "Definition of categories of speech transmission quality", September '99

[15] Miroslaw Narbutt, Andrew Kelly, Liam Murphy, Philip Perry, "Adaptive VoIP Playout Scheduling: Assessing User Satisfaction,"IEEE Internet Computing Magazine, vol. 09, no. 4, July/August 2005.

[16] Miroslaw Narbutt, Mark Davis, "Assessing the Quality of VoIP Transmission Affected by Playout Buffer Scheme," Proc. of the ETSI/IEE Measurement of Speach and Audio Quality in Networks Conference 2005 (MESAQIN 2005), June 2005, Prague.

[17] Miroslaw Narbutt, Mark Davis, "An Assessment of the Audio Codec Performance in Voice over WLAN (VoWLAN) Systems," Proc. of the International Conference on Mobile and Ubiquitous Systems: Networking and Services, (MOBIQUITOUS 2005), July 2005, San Diego.

[18] RTPtools: http://www.cs.columbia.edu/IRT/software/rtptools

[19] ITU-T Recommendation P.59, “Artificial conversational speech", March 1993

[20] R. Ramjee, J. Kurose, D. Towsley, and H. Schulzrinne, "Adaptive playout mechanisms for packetized audio applications in wide-area networks", Proc. of the IEEE Infocom, Toronto, 1994 
encoding G711 (80Bytes/10ms)
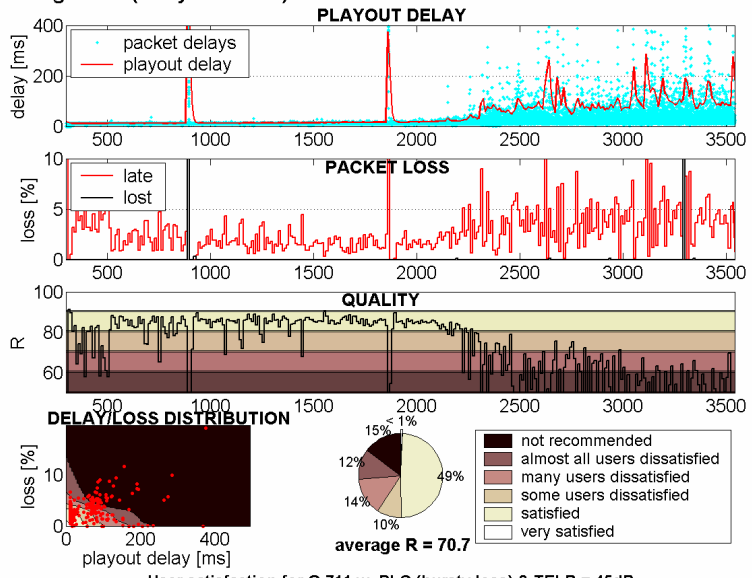

User satisfaction for G.711 w. PLC (bursty loss) \& TELR $=45 \mathrm{~dB}$

Figure 13. Call quality and user satisfaction with CBR traffic (G.711, 80bytes/10ms) -wired side
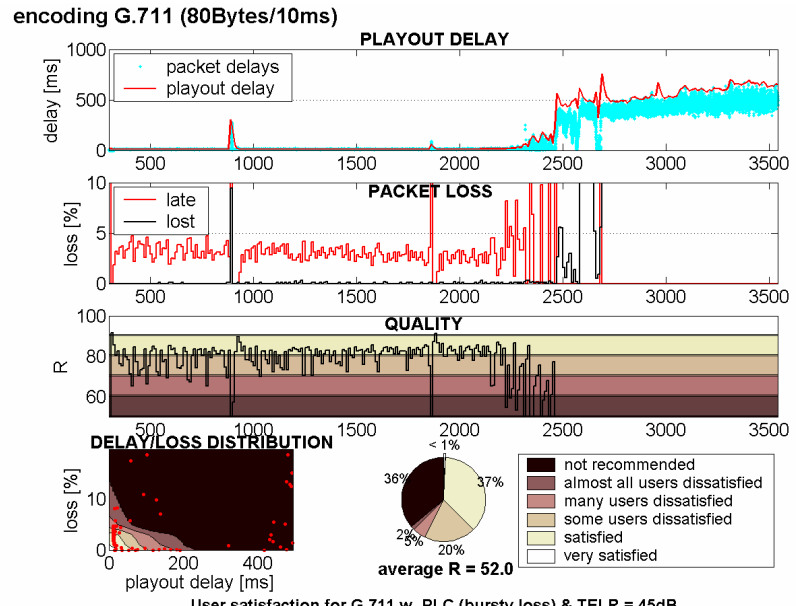

Figure 14. Call quality and user satisfaction with CBR traffic (G.711, 80bytes/10ms) -wireless side

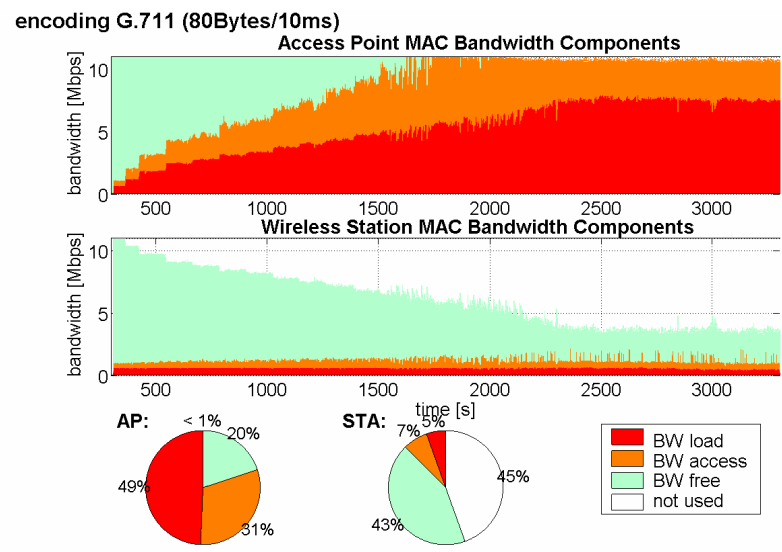

Figure 15. MAC bandwidth utilization with CBR traffic (G.711 80bytes/10ms) at the access point and the wireless station encoding G.711 (80Bytes/10ms)
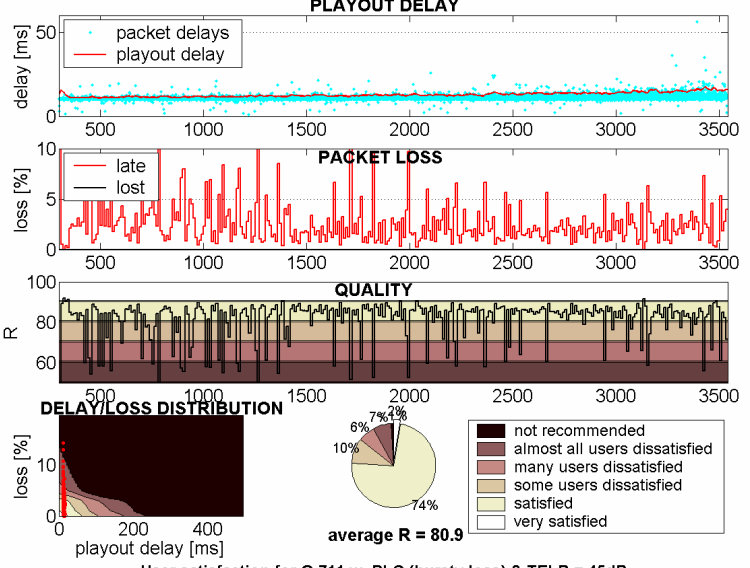

User satisfaction for G.711 w. PLC (bursty loss) \& TELR $=45 \mathrm{~dB}$

Figure 16. Call quality and user satisfaction with ON-OFF traffic (G.711, 80bytes/10ms) -wired side

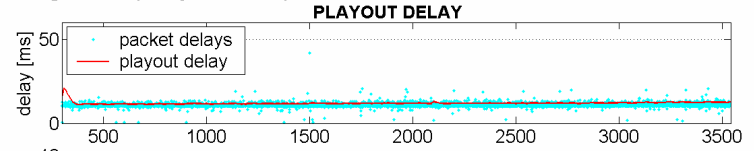

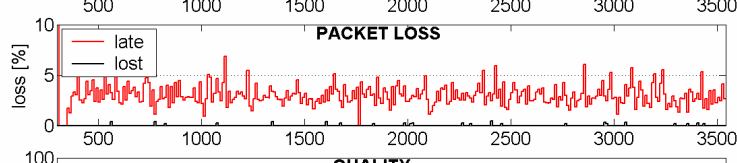

100 QUALITY

×

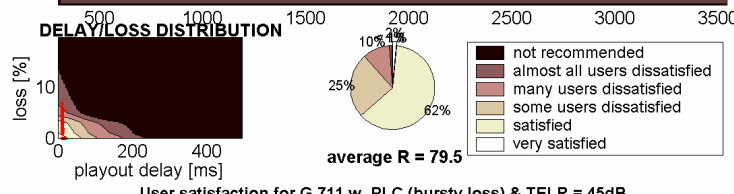

Figure 17. Call quality and user satisfaction with ON-OFF traffic (G.711, 80bytes/10ms) -wireless side

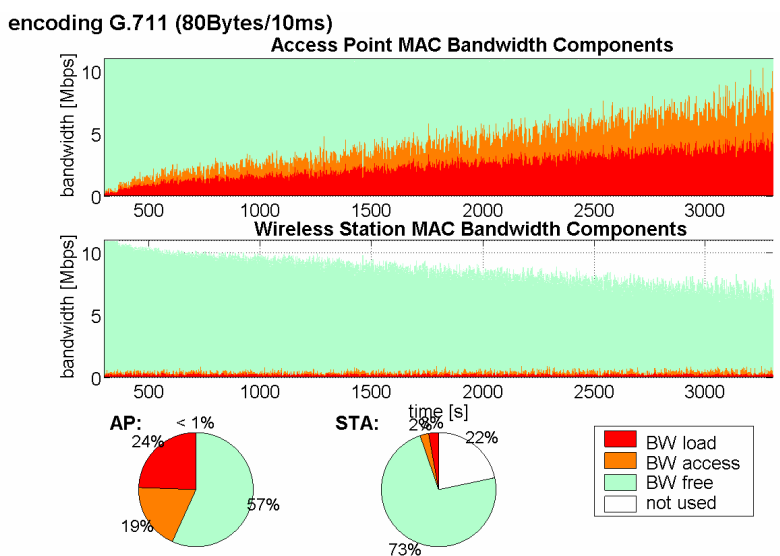

Figure 18. MAC bandwidth utilization with ON-OFF traffic (G.711 80bytes/10ms) at the access point and the wireless station 
encoding G.711 (160Bytes/20ms)
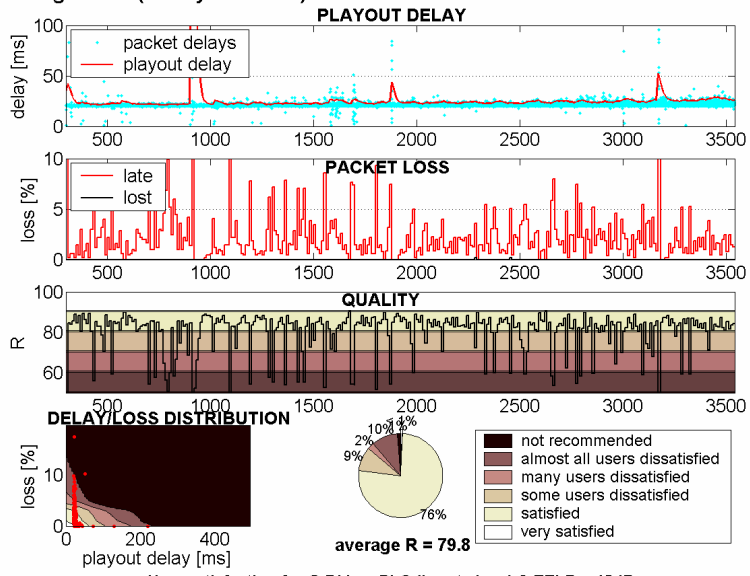

User satisfaction for $G .711$ w. PLC (bursty loss) \& TELR $=45$ dB

Figure 19. Call quality and user satisfaction with CBR traffic (G.711, 160bytes/20ms) - wired side

encoding G.711 (160Bytes/20ms)
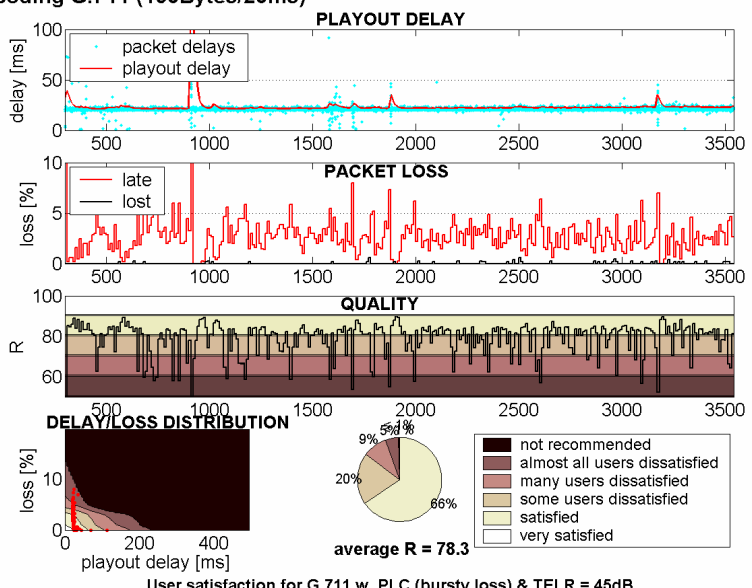

Figure 20. Call quality and user satisfaction with CBR traffic (G.711, 160bytes/20ms) - wireless side

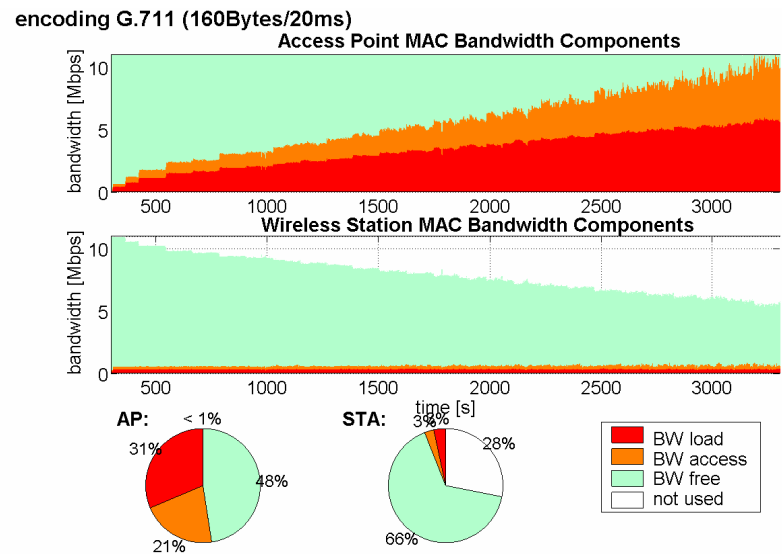

Figure 21. MAC bandwidth utilization with CBR traffic (G.711 160bytes/20ms) at the access point and the wireless station encoding G.711 (160Bytes/20ms)
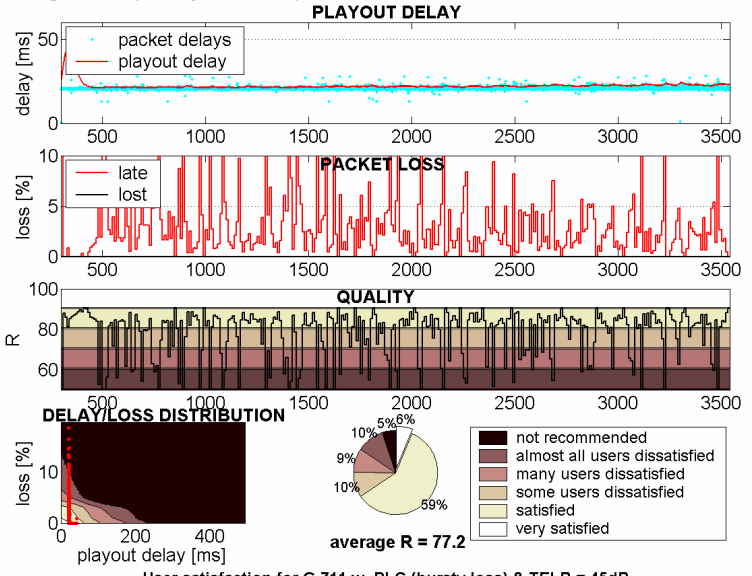

User satisfaction for G.711 w. PLC (bursty loss) \& TELR $=45 \mathrm{~dB}$

Figure 22. Call quality and user satisfaction with ON-OFF traffic (G.711, 160bytes/20ms)-wired side

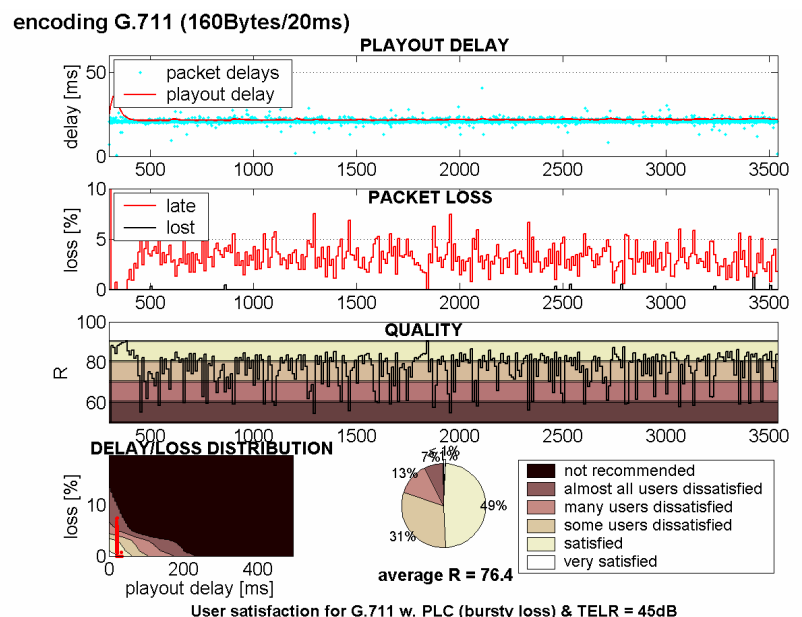

Figure 23. Call quality and user satisfaction with ON-OFF traffic (G.711, 160bytes/20ms) -wireless side

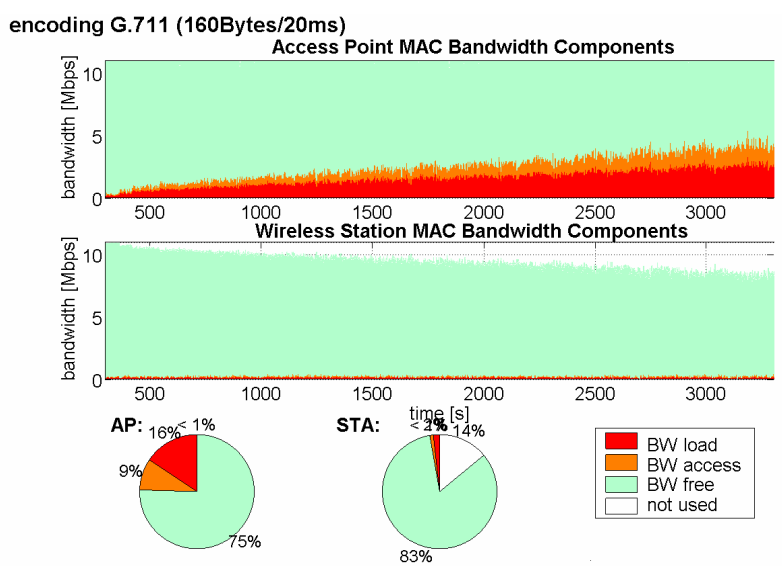

Figure 24. MAC bandwidth utilization with ON-OFF traffic (G.711 160bytes/20ms) at the access point and the wireless station 
encoding G.723.1 (24Bytes/30ms)
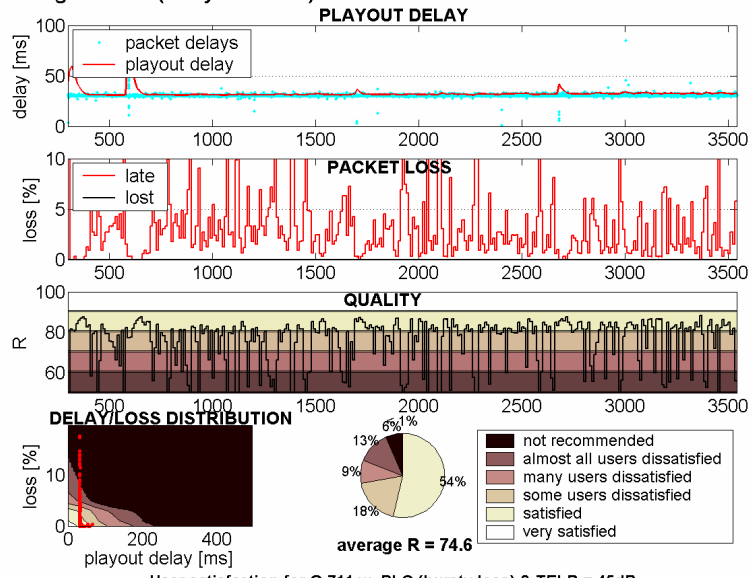

User satisfaction for $G .711$ w. PLC (bursty loss) \& TELR $=45$ dB

Figure 25. Call quality and user satisfaction with CBR traffic (G.723.1, 24bytes/30ms) - wired side

encoding G.723.1 (24Bytes/30ms)
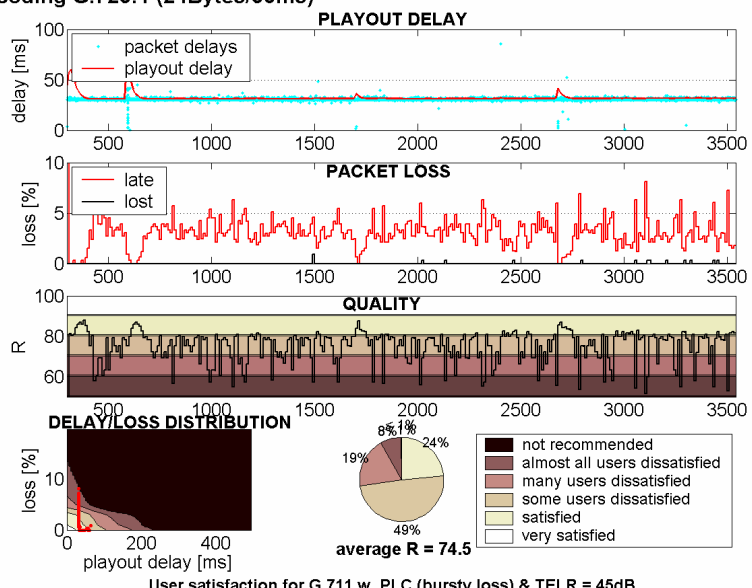

Figure 26. Call quality and user satisfaction with CBR traffic (G.723.1, 24bytes/30ms) - wireless side

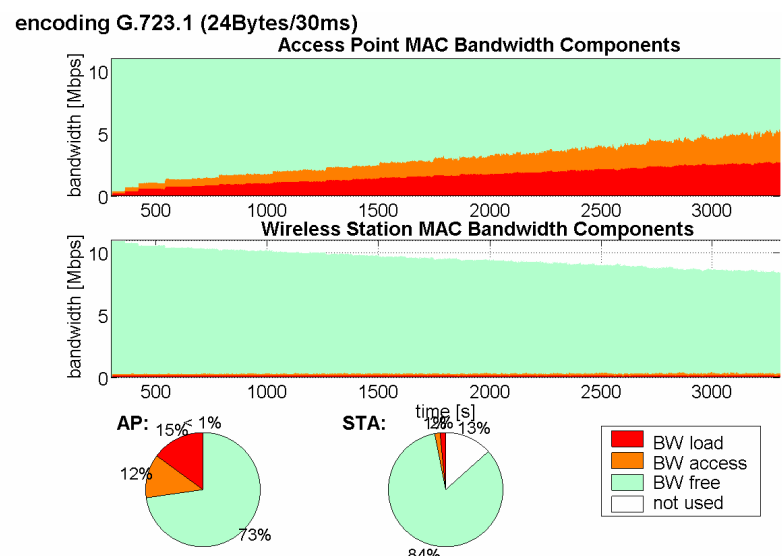

Figure 27. MAC bandwidth utilization with CBR traffic (G.723.1 24bytes/30ms) at the access point and the wireless station encoding G.723.1 (24Bytes/30ms)
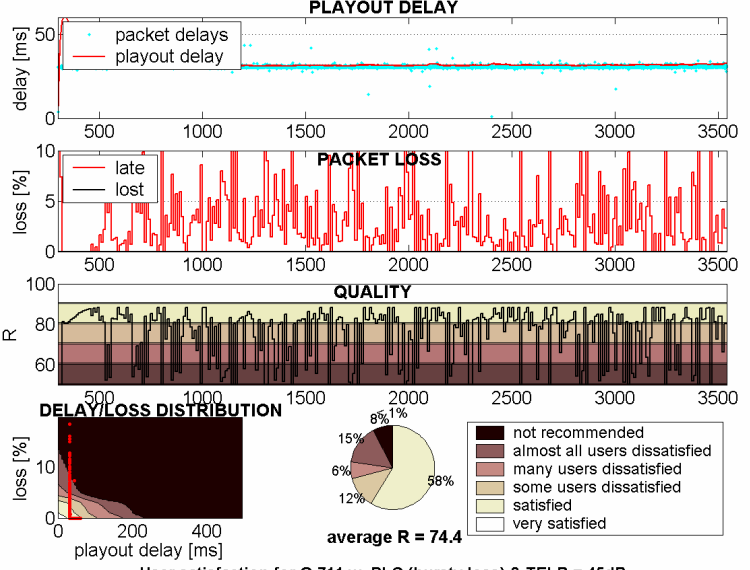

User satisfaction for G.711 w. PLC (bursty loss) \& TELR $=45 \mathrm{~dB}$

Figure 28. Call quality and user satisfaction with ON-OFF traffic (G.723.1, 24bytes/30ms) - wired side

encoding G.723.1 (24Bytes/30ms)
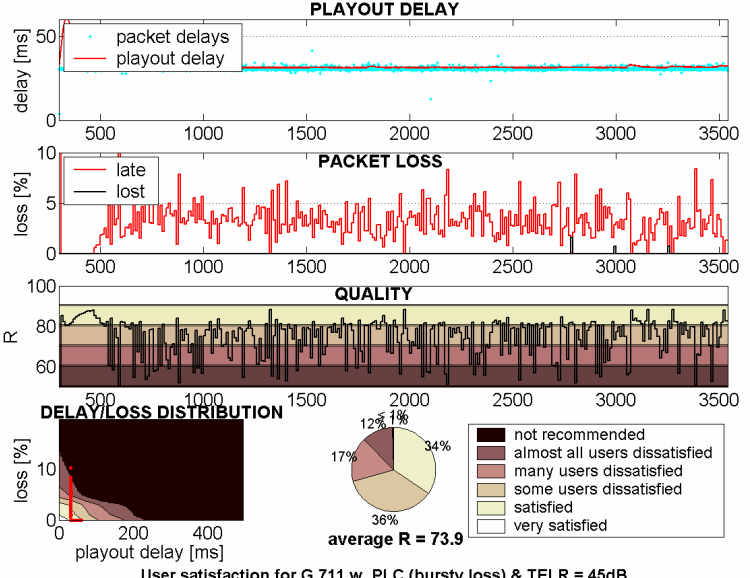

Figure 29. Call quality and user satisfaction with ON-OFF traffic (G.723.1, 24bytes/30ms) - wireless side

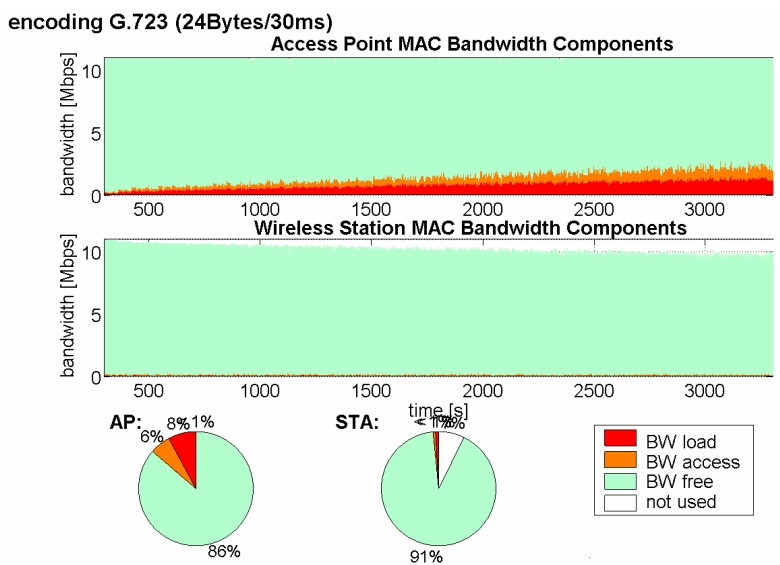

Figure 30. MAC bandwidth utilization with ON-OFF traffic (G.723.1 24bytes/30ms) at the access point and the wireless station 
encoding G.729 (10Bytes/10ms)
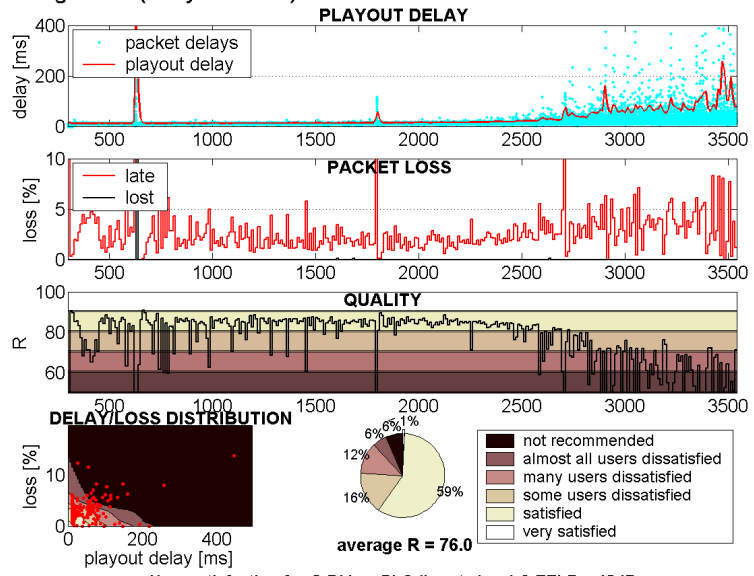

User satisfaction for G.711 w. PLC (bursty loss) \& TELR $=45 \mathrm{~dB}$

Figure 31. Call quality and user satisfaction with CBR traffic (G.729, 10bytes/10ms) - wired side
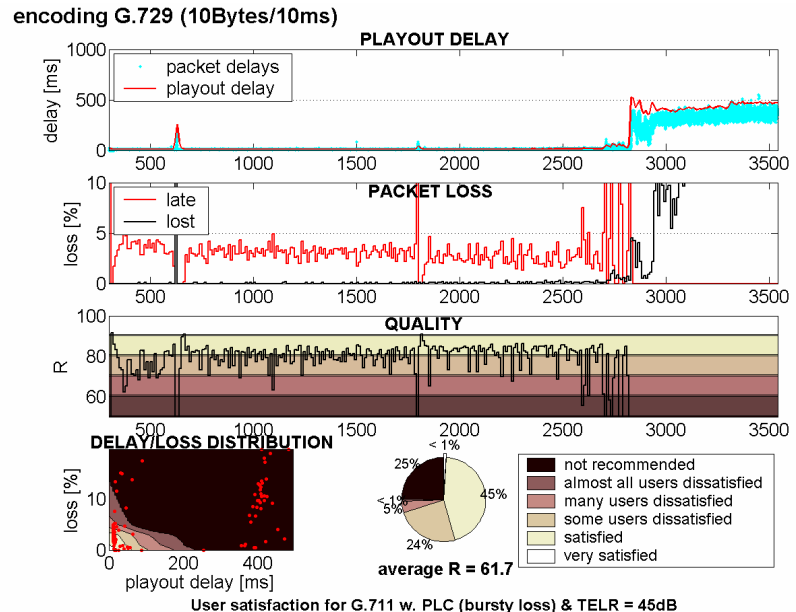

Figure 32. Call quality and user satisfaction with CBR traffic (G.729, 10bytes/10ms) - wireless side

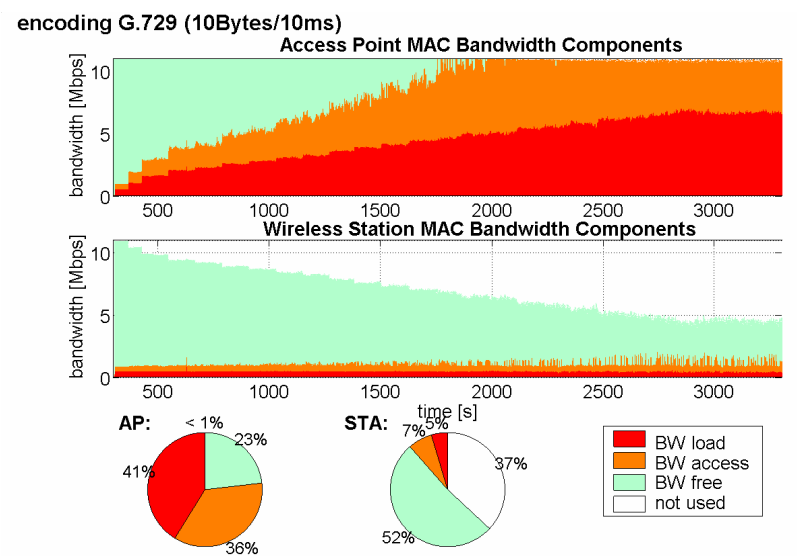

Figure 33. MAC bandwidth utilization with CBR traffic (G.729 10bytes/10ms) at the access point and the wireless station encoding G.729 (10Bytes/10ms)
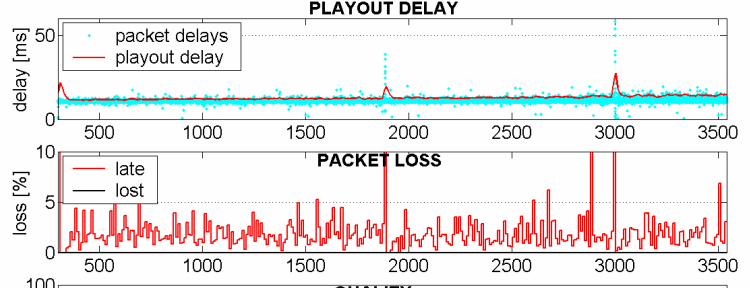

$100 \quad 500 \quad 1000 \quad 1500$ QUALITY $2000 \quad 2500 \quad 3000$

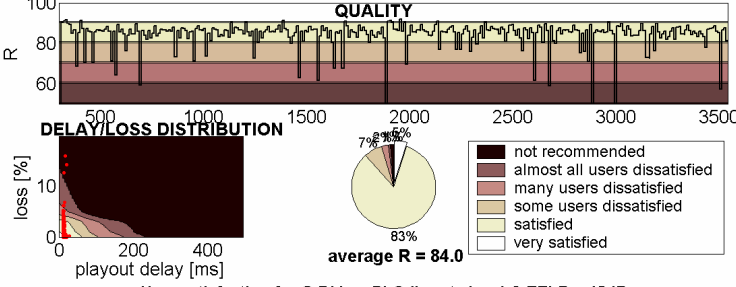

playout delay [ms]

Figure 34. Call quality and user satisfaction with ON-OFF traffic (G.729, 10bytes/10ms) - wired side

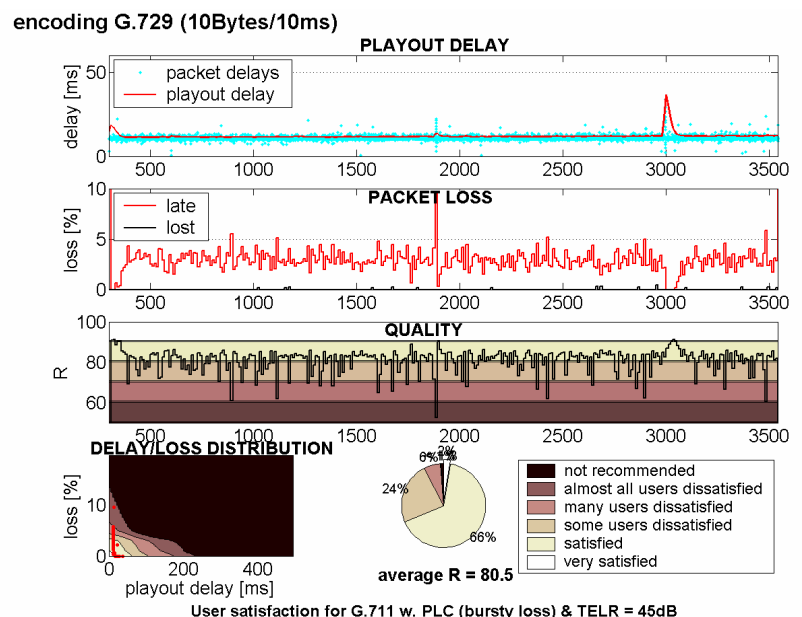

Figure 35. Call quality and user satisfaction with ON-OFF traffic (G.729, 10bytes/10ms) - wireless side

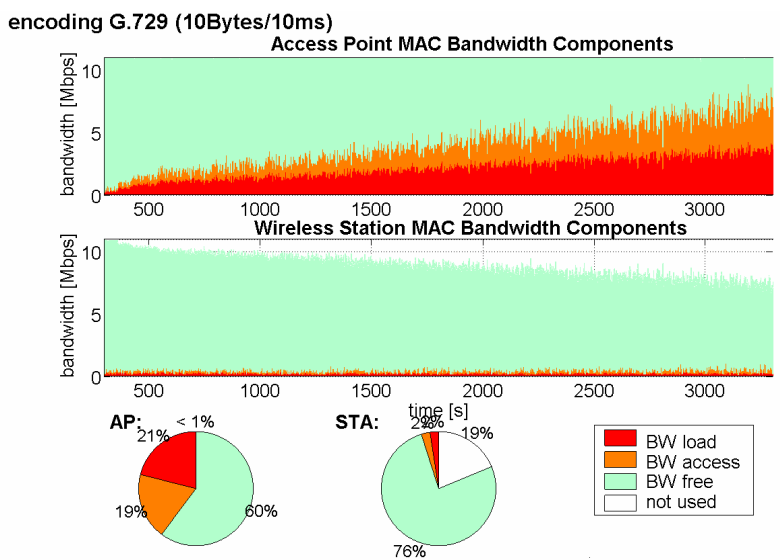

Figure 36. MAC bandwidth utilization with ON-OFF traffic (G.729 10bytes/10ms) at the access point and the wireless station 